Annales Geophysicae (2002) 20: 729-740 (c) European Geophysical Society 2002

\title{
Instabilities of continuously stratified zonal equatorial jets in a periodic channel model
}

\author{
S. Masina \\ Istituto Nazionale di Geofisica e Vulcanologia, I-00143, Roma, Italy \\ Received: 27 August 2001 - Revised: 7 January 2002 - Accepted: 10 January 2002
}

\begin{abstract}
Several numerical experiments are performed in a nonlinear, multi-level periodic channel model centered on the equator with different zonally uniform background flows which resemble the South Equatorial Current (SEC). Analysis of the simulations focuses on identifying stability criteria for a continuously stratified fluid near the equator. A $90 \mathrm{~m}$ deep frontal layer is required to destabilize a zonally uniform, $10^{\circ}$ wide, westward surface jet that is symmetric about the equator and has a maximum velocity of $100 \mathrm{~cm} / \mathrm{s}$. In this case, the phase velocity of the excited unstable waves is very similar to the phase speed of the Tropical Instability Waves (TIWs) observed in the eastern Pacific Ocean. The vertical scale of the baroclinic waves corresponds to the frontal layer depth and their phase speed increases as the vertical shear of the jet is doubled. When the westward surface parabolic jet is made asymmetric about the equator, in order to simulate more realistically the structure of the SEC in the eastern Pacific, two kinds of instability are generated. The oscillations that grow north of the equator have a baroclinic nature, while those generated on and very close to the equator have a barotropic nature.

This study shows that the potential for baroclinic instability in the equatorial region can be as large as at mid-latitudes, if the tendency of isotherms to have a smaller slope for a given zonal velocity, when the Coriolis parameter vanishes, is compensated for by the wind effect.
\end{abstract}

Key words. Oceanography: general (equatorial oceanography; numerical modeling) - Oceanography: physics (fronts and jets)

\section{Introduction}

After the pioneering work of Eady (1949) on baroclinic instability at mid-latitudes under the quasi-geostrophic approximation, Stone $(1966,1970)$ used Eady's model to study the

Correspondence to: S. Masina (masina@ingv.it) baroclinic instability in the ageostrophic regime. Stone's results confirm that baroclinic instability is possible also under ageostrophic conditions; however, so far, this conclusion has not been demonstrated for the equatorial case. Ripa (1991) showed that stability conditions for ageostrophic flows at the equator are more easily violated in a primitive-equation multi-layer model as the number of layers increases. Recent results (Benilov and Sakov, 1999) showed that if realistic velocity and density profiles are used instead of the linear approximation baroclinic instability becomes up to 6 times stronger in quasi-geostrophic flows. These conclusions need to be proven for ageostrophic flows. In general, stability conditions for an equatorial continuous stratified model have not yet been obtained and the analytical approach to determine such criteria remains one of the most challenging unresolved problems related to equatorial dynamics. In this study, we will investigate numerically the hypothesis of having baroclinic instability close to the equator in a continuously stratified fluid through the analysis of several experiments that we have conducted with a channel model centered on the equator with various mean states.

Additionally, these experiments have proven invaluable in identifying the main processes that develop the Tropical Instability Waves (TIWs) in the Pacific Ocean at a 20-30 day period and about a $1000 \mathrm{~km}$ wavelength (Masina et al., 1999). In a $1 \frac{1}{2}$-layer model, a zonal current profile similar to the equatorial system in the Pacific and in the Atlantic is barotropically unstable to westward waves, with a period of about 1 month and a zonal wavelength of about $1000 \mathrm{~km}$ (Philander, 1978). However, Semtner and Holland (1980) and Cox (1980) found an instability with similar wavelength and period in a numerical simulation of a continuously stratified ocean and demonstrated that baroclinic instability represents a significant energy source for the growing unstable waves. In this work, we investigate the possible mean-flow configurations that show some characteristics similar to those of the equatorial Pacific and that are unstable to waves that have a spatial structure and a temporal periodicity similar to those observed (Legeckis, 1977; Qiao and Weisberg, 1995; 
Kennan and Flament, 2000) and simulated (Masina and Philander, 1999; McCreary and Yu, 1992; Donohue and Wimbush, 1998; Proehl, 1998). Emphasis is placed on the effects of stratification on the instability waves. In particular, the consequence of the presence of a frontal layer on the mean flow stability will be investigated.

In Sect. 2, we describe the channel model that has been used in this study. A series of experiments has been carried out to test the stability of different mean flow configurations. We will first outline here the motivation behind each set of experiments. The first mean flow that we used as an initial condition is a westward surface jet $U(y, z)$ centered on the equator (Sect. 3). With this mean flow configuration we performed two sets of experiments; the first set of experiments consists of five sensitivity experiments performed in order to test the dependence of the wave on the frontal layer depth and on stratification (Sect. 3.1), and the second set consists of three experiments that have been integrated in time in order to examine how the speed of the unstable mean state jet affects the phase speed of the wave (Sect. 3.4). The second mean flow that we used is a surface westward jet asymmetric about the equator (Sect. 4) and which resembles the SEC in the eastern Pacific. Finally, in Sect. 5, we summarize and discuss the main results.

\section{Description of the periodic channel model}

The multi-level primitive equations Modular Ocean Model (MOM) (Pacanowski, Dixon and Rosati, 1993) produced at the Geophysical Fluid Dynamics Laboratory (GFDL) is applied to a zonal channel domain centered on the equator and bounded latitudinally by solid walls at $10^{\circ} \mathrm{S}$ and $10^{\circ} \mathrm{N}$. Cyclic boundary conditions are applied at the eastern and western ends of the channel. The zonal length of the domain is chosen to be $10^{\circ}$ of longitude, which is representative of the average wavelength of the unstable waves observed in the Pacific Ocean. The maximum wavelength of waves that can be excited in a channel model is equal to the zonal length of the domain. Therefore, the choice of domain length is crucial in such experiments and we have chosen it such that all the observed wavelengths $\leq 1100 \mathrm{~km}$ may be captured in the experiments to be described here. An experiment performed in a longer domain $\left(100^{\circ}\right)$ showed that the wavelength of the fastest growing waves is the same as in the $10^{\circ}$ long domain, but that the amplitude of the unstable waves is smaller. The latter effect is probably due to the negative interference of modes with different wavelengths which can grow in a longer domain. The depth of the basin is $3000 \mathrm{~m}$ and 18 levels are unevenly distributed in such a way that the vertical resolution is $10 \mathrm{~m}$ in the uppermost $100 \mathrm{~m}$ and it increases with depth below this level. The resolution is $\frac{1}{2}^{\circ}$ in the zonal direction and $\frac{1}{4}^{\circ}$ in the meridional direction. Neither Newtonian damping nor sponge boundaries are used on the lateral walls; rather, free-slip conditions are imposed. A biharmonic lateral mixing scheme is used with horizontal viscosity and diffusivity coefficients constant and equal to $5.0 \times 10^{20} \mathrm{~cm}^{4} / \mathrm{s}$. The
Pacanowski and Philander (1981) vertical mixing scheme is applied. An experiment with decreased zonal and meridional resolution $\left(\Delta x=1^{\circ}, \Delta y=\frac{1}{3}^{\circ}\right)$ but the same mixing coefficients, has demonstrated that the coarser resolution induces more viscosity in the system and tends to stabilize the mean flow.

In the eastern tropical Pacific, density stratification is mainly governed by temperature and this allows us to neglect salinity effects and to simplify the equation of state. Density is calculated from temperature through the linear equation of state,

$\rho=\rho_{0}(1-\alpha T)$

where the thermal expansion coefficient, $\alpha$, is fixed at a typical value of $4.3 \times 10^{-4}{ }^{\circ} \mathrm{C}^{-1}$; the reference density $\rho_{0}=1 \mathrm{gr} / \mathrm{cm}^{3}$.

The mean flow is strictly zonal at the initial time $t=0$ and the mean temperature $T_{g}$ is calculated from it via the thermal wind equation (see next section). The model grid is chosen to ensure that there are no temperature grid points directly on the equator and consequently, no singular situation arises (Cox, 1980). The mean state zonal velocity is described by an analytical function with latitudinal and vertical dependence, as described in the following section. The functions that we employ have been chosen to simulate realistic, even if simplified, aspects of the currents in the equatorial Pacific.

The velocity and temperature fields are decomposed into mean state and perturbation quantities according to:

$u=\bar{U}(y, z, t)+u^{\prime}(x, y, z, t)$,

$v=\bar{V}(y, z, t)+v^{\prime}(x, y, z, t)$,

$T=\bar{T}(y, z, t)+T^{\prime}(x, y, z, t)$,

where the primes indicate a fluctuation about the zonal mean. The initial conditions on primed quantities are

$u^{\prime}=T^{\prime}=0$,

$v^{\prime}=R(x, y), \quad 0 \geq z \geq-113 \mathrm{~m}$,

$v^{\prime}=0, \quad-113>z \geq-3000 \mathrm{~m}$,

where $R$ is a randomly generated number distribution with limits of $\pm 9 \mathrm{~cm} / \mathrm{s}$; these limits have been chosen to be less than $10 \%$ of the maximum speed of the mean flow. A random initial perturbation is chosen instead of an oscillating function of the form $\exp (k x-\omega t)$, in order to introduce all possible frequencies of the system. Admissible wavelengths are constrained by the zonal length of the domain, as we explained above. This same technique has been used by Orlanski and Cox (1973) and Cox (1980) for similar experiments.

The list of all the experiments performed with the channel model is displayed in Table 1. 
Table 1. List of experiments. First column: experiment names. Second column: parameter used in Eq. (8) in cm/s. Third column: frontal layer depth in meters. Fourth column: vertical temperature gradient inside the frontal layer in ${ }^{\circ} \mathrm{C} / \mathrm{cm}$. Fifth column: zonal length of the domain in degrees

\begin{tabular}{ccccc}
\hline EXP & $\begin{array}{c}U_{0} \\
{[\mathrm{~cm} / \mathrm{s}]}\end{array}$ & $\begin{array}{c}\text { Frontal Layer } \\
\text { Depth }[\mathrm{m}]\end{array}$ & $\begin{array}{c}\frac{\partial T}{\partial z} \text { in M.L. } \\
{\left[{ }^{\circ} \mathrm{C} / \mathrm{cm}\right]}\end{array}$ & $\mathrm{L}$ \\
\hline EXP 1 & -100 & 0 & geos. & $10^{\circ}$ \\
EXP 2 & -100 & 60 & 0 & $10^{\circ}$ \\
EXP 3 & -100 & 90 & 0 & $10^{\circ}$ \\
EXP 4 & -100 & 90 & $10^{-4}$ & $10^{\circ}$ \\
EXP 5 & -100 & 90 & $5 \times 10^{-5}$ & $10^{\circ}$ \\
EXP 6 & -50 & 90 & 0 & $15^{\circ}$ \\
EXP 7 & -100 & 90 & 0 & $15^{\circ}$ \\
EXP 8 & -200 & 90 & 0 & $15^{\circ}$ \\
EXP 9 & -100 & 0 & geos. & $10^{\circ}$ \\
\hline
\end{tabular}

\section{Mean flow I: a surface westward jet centered on the equator}

The analytical expression that is used to calculate the initial condition for the mean flow $U(\theta, z)$ in the first 8 experiments is

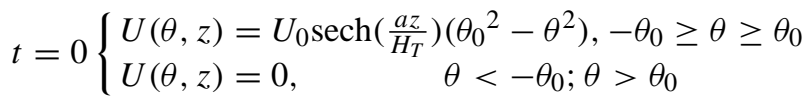

in which $\theta$ is latitude (in degrees), $\theta_{0}=5^{\circ}, U_{0}$ varies from $-50 \mathrm{~cm} / \mathrm{s}$ to $-200 \mathrm{~cm} / \mathrm{s}$ in the experiments, $H_{T}=-3000 \mathrm{~m}$ and $a=60$. An estimate of the second derivative in latitude of the flow at $t=0, U_{y y}$ shows that this term is 3 orders of magnitude smaller than $\beta$ and, therefore, the latitudinal gradient of the potential vorticity is positive at all latitudes. At mid-latitudes, this condition would be sufficient to preclude the possibility of barotropic instability (Kuo, 1949). In the equatorial region, however, this condition is not sufficient, since horizontal divergence is a crucial factor in equatorial dynamics. Lipps (1963) first introduced divergence effects in the stability condition for a quasi-geostrophic flow on a $\beta$-plane in a $1 \frac{1}{2}$-layer model. If one does not make the $\mathrm{Q}-\mathrm{G}$ approximation, it is possible to derive a stability condition that applies to strong equatorial zonal currents in the reduced gravity model approximation (McPhaden and Ripa, 1990). If the meridional gradient of the potential vorticity

$Q_{y}=\left[\beta-U_{y y}+U f\left(f-U_{y}\right) / g^{\prime} H\right] / H$

is of only one sign, then the flow is barotropically stable. This stability condition shows that in the equatorial region, as well as at middle latitudes, both $\beta$ and divergence, $\frac{U f\left(f-U_{y}\right)}{g^{\prime} H}$, can stabilize eastward jets, yet destabilize westward jets, to barotropic instability (Philander, 1976). No other stability criteria are available for a continuously stratified model in the equatorial region. An estimate of the term $\frac{U f\left(f-U_{y}\right)}{g^{\prime} H}$ in
Eq. (9) for a first baroclinic mode shows that is always positive and of the same order of magnitude of $\beta$ for the mean flow defined in Eq. (8). This supports the conclusion that the mean state is barotropically stable at $t=0$. For other possible and even more realistic mean flow configurations, such as a double jet resembling the westward SEC and the eastward North Equatorial Countercurrent, $Q_{y}$ can change sign in latitude and the flow can be barotropically unstable.

The thermal wind equation is

$f U_{z}=-g \alpha T_{g y}$,

where $g$ is gravity and $\alpha$ is the thermal expansion coefficient, as defined in the equation of state (1). $f$ is the Coriolis parameter given by

$f=2 \omega \sin \theta \simeq 2 \omega \frac{y}{r}$,

where $\omega$ and $r$ are the angular velocity and the radius of the earth, respectively.

Combining Eqs. (8) and (10) and using the approximation $y \simeq \theta r$ valid for small $\theta$, we can obtain an analytical expression for the temperature $T_{g}$,

$T_{g}(y, z)=T_{0}(z)-\frac{2 \omega a U_{0}}{g \alpha r^{3} H} \frac{\sinh \left(\frac{a z}{H}\right)}{\cos ^{2} h\left(\frac{a z}{H}\right)}\left(-\frac{1}{4} y^{4}+\frac{1}{2} y^{2} y_{0}^{2}\right)$.

$T_{0}(z)$ is chosen to be a typical vertical temperature profile that is representative of that occurring in the eastern Pacific.

3.1 EXP 1, EXP 2, EXP 3, EXP 4, EXP 5: initial conditions and results

In this first set of experiments, the parameter $U_{0}$ of the mean flow has been kept fixed at the value of $-100 \mathrm{~cm} / \mathrm{s}$. The zonal velocity and temperature fields used as the initial condition for EXP 1 are shown in Fig. 1a (only the upper 12 model levels are shown for clarity).

EXP 2 is identical to EXP 1, with the exception that a $60 \mathrm{~m}$ deep frontal layer with zero stratification $\left(N^{2}=0\right)$ is introduced in the thermal structure $T_{g}(y, z)$ at all latitudes. In EXP 3 the depth of the frontal layer is increased to $90 \mathrm{~m}$. The initial conditions used for $U(y, z)$ and $T(y, z)$ (omitting the subscript) in EXP 2 and in EXP 3 are shown in Fig. 1b and Fig. 1c, respectively. In EXP 4 and EXP 5, we introduce a small constant vertical temperature shear $T_{z}$ in the $90 \mathrm{~m}$ deep frontal layer of EXP 3, in order to study the case with $N^{2} \neq 0$. In EXP 4, the temperature difference $\nabla T$ over the depth of the frontal layer is $0.9^{\circ} \mathrm{C}$, while in EXP $5 \nabla T$ is decreased to $0.45^{\circ} \mathrm{C}$ over the same depth. The initial condition used for EXP 5 is shown in Fig. 1d.

We observe that the ageostrophic effects introduced in the channel model by forcing the presence of a frontal layer or of stratification much smaller than that desired by geostrophy excites spurious small amplitude disturbances at the beginning of the numerical integration. Therefore, the experiments have been repeated using a zonally uniform mean flow $U(y, z)$, which is in balance with the new temperature field 

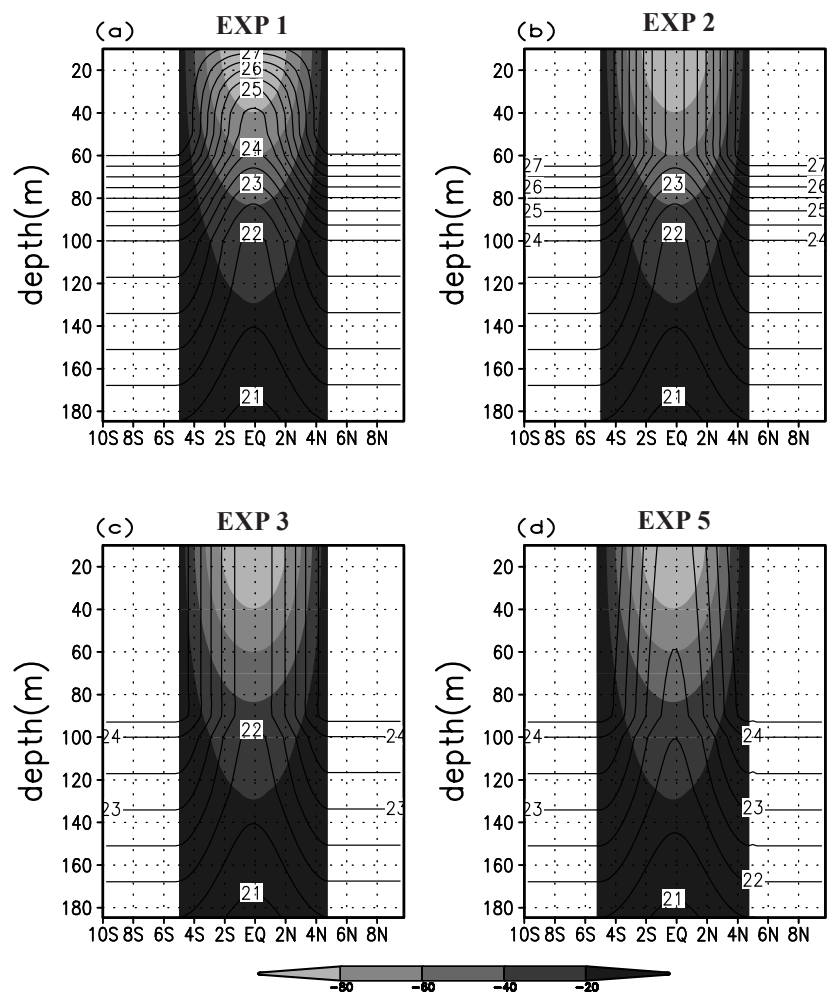

Fig. 1. Depth-latitude sections of zonally uniform horizontal velocity (shaded) and temperature field (contour) used as the initial condition for: (a) EXP 1, (b) EXP 2, (c) EXP 3 and (d) EXP 5. Contour interval is $0.5^{\circ} \mathrm{C}$ for temperature and $20 \mathrm{~cm} / \mathrm{s}$ for velocity.

after the modification imposed on the frontal layer. The results do not show any significant differences with respect to the experiments performed with the thermally unbalanced flow. These results induce us to believe that the dynamical evolution of the simulation is not affected by the introduction of an unbalanced frontal layer at $t=0$.

EXP 1 is stable, while EXP 3, EXP 4 and EXP 5 generate very similar unstable oscillations in the equatorial band between $2^{\circ} \mathrm{S}$ and $2^{\circ} \mathrm{N}$. In Fig. 2 we show the surface perturbation meridional velocity (i.e. the deviation from the zonal mean) for EXP 3 as a function of time and latitude.

Oscillations with a period of 23 days are evident particularly south of the equator after a couple of weeks of integration. The mean flow provides energy to the oscillations in such a way that they can grow linearly in time. The oscillations south of the equator reach a maximum amplitude of $8 \mathrm{~cm} / \mathrm{s}$ after two months of integration. At this time they are saturated and never reach a chaotic behaviour. The instability waves generated in EXP 3 have a wavelength that is exactly the length of the model domain, i.e. $10^{\circ}$ of longitude, corresponding to $\simeq 1100 \mathrm{~km}$. The meridional scale of the waves is $\simeq 2^{\circ}$ and corresponds approximately to one equatorial Rossby radius of deformation.

A picture (not shown) similar to Fig. 2 is obtained for EXP 2, even if in this case the amplitude of the instability is much weaker. Oscillations with structure similar to

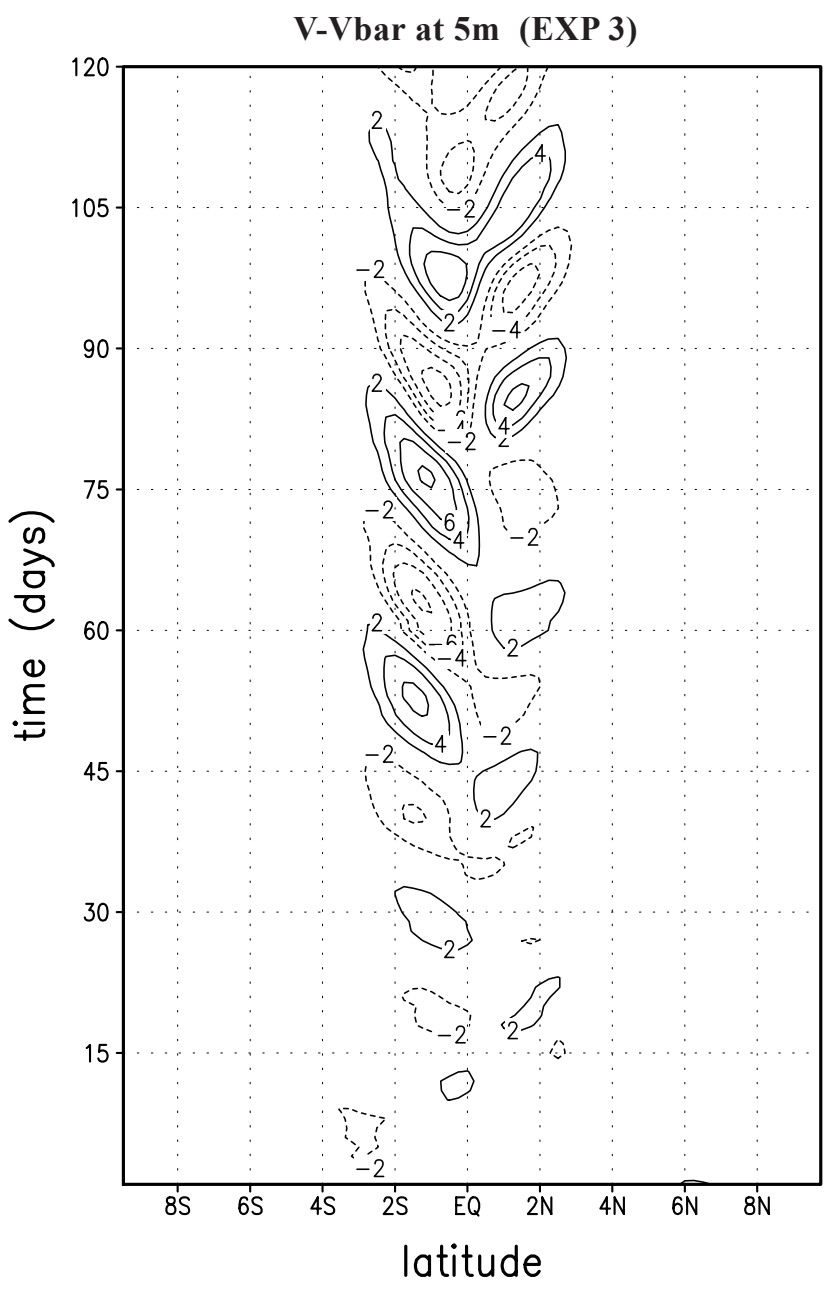

Fig. 2. Time-latitude diagram of perturbation meridional velocity at $5 \mathrm{~m}$ for EXP 3. Contour interval is $2 \mathrm{~cm} / \mathrm{s}$.

the unstable waves generated in EXP 3 are observed also in this case. The oscillations are initially excited by the random $v^{\prime}$ initial condition and geostrophic adjustment, but then decay. The global eddy kinetic energy of the system in EXP 2 decays instead of exponentially growing, indicating that the mean flow of EXP 2 is stable. Oscillations similar to those generated in EXP 3 arise both in EXP 4 and EXP 5. Their wavelength is $\simeq 1100 \mathrm{~km}$ and their period is 21 days. Not only the structure but also the amplitude of the oscillations is very similar between EXP 3 and EXP 5, while in EXP 4 the perturbations are smaller in magnitude, suggesting a weaker instability.

\subsection{The effect of stratification versus that of a frontal layer}

The most striking result that we derive from the comparison of EXP 1, EXP 2 and EXP 3 is that the presence of a frontal layer in the region where the mean flow $U(y, z) \neq 0$ destabilizes the westward jet and induces instability waves similar to the oscillations observed in the eastern Pacific Ocean. EXP 1 is stably stratified at all depths. The geostrophically balanced 
initial temperature for EXP 1 does not show any meridional temperature gradient at the surface of the ocean, because the isotherms flatten at the equator as a result of the vanishing of the Coriolis parameter (see Fig. 1a) and the thermal wind equation (Eq. 10), and we showed that this experiment is stable. EXP 2 and EXP 3 differ from EXP 1 only in the presence of a frontal layer. We showed that the depth of the frontal layer is a crucial parameter in the instability process. EXP 2, which has a $60 \mathrm{~m}$ deep frontal layer, is stable but if we increase the frontal layer depth to $90 \mathrm{~m}$, as we did in EXP 3, the mean flow becomes unstable. The effect of the frontal layer is to reduce the stratification to zero and to introduce a meridional temperature gradient at the ocean surface. In the channel model, the artificial frontal layer simulates a wind forcing that induces upwelling at the equator and establishes a meridional temperature gradient, from the thermocline up to the surface, that supplies potential energy available to be used by the instability growth. Furthermore, for a fluid with zero stratification, the available potential energy is proportional to the depth of the fluid (see Eq. 7.8.10 in Gill, 1982). An estimate shows that the available potential energy of the unstable EXP 3 is approximally three times larger than the corresponding quantity in EXP 2, which is stable.

We have shown that the introduction of a frontal layer with zero stratification $\left(N^{2}=0\right)$ destabilizes a mean flow system. However, we have also demonstrated that the condition $N^{2}=0$ is not strictly necessary to render a system unstable. In fact, in EXP 4 and EXP 5 we have shown that even with a mean stratification different from zero $\left(N^{2} \neq 0\right)$, the system can be unstable. In EXP 4 and EXP 5, the stratification is not completely reduced to zero, but a meridional temperature gradient is still present at the ocean surface. For the same temperature $T$, the system with the smaller vertical temperature gradient $T_{z}$ has more mean potential energy because, for a system with mean stratification, the mean potential energy is proportional to the ratio $\frac{T}{T}$. An estimate of the critical vertical temperature gradient is possible if we consider the Burger number condition for baroclinic instability (e.g. Orlanski, 1986). The Burger number is defined as

$B=R_{0}^{2} R_{i}$,

where $R_{0}$ is the Rossby number $\left(R_{0}=\frac{U}{f L}\right)$ and $R_{i}$ $\left(N^{2} H^{2} / U^{2}\right)$ is the Richardson number. The Burger number defines the ratio of the slope of particle trajectories $(w / v)$ to the slope of the isotherms $\left(T_{y} / T_{z}\right)$ in a $\mathrm{Q}-\mathrm{G}$ regime. However this result can be extended to the nongeostrophic regime up to $R_{0} \simeq 1$ (Orlanski and Cox, 1973). For baroclinic instability to be possible, it is required that the Burger number

$B=R_{0}^{2} R_{i} \leq 1$.

In the quasi-geostrophic limit, $R_{0} \rightarrow 0, R_{i}$ can be very large which implies that the mean stratification of the system can be very strong. On the contrary, when $R_{0} \simeq 1$ and nongeostrophic effects have to be taken into consideration, the Burger number condition for baroclinic instability requires that $R_{i} \leq 1$. This imposes a critical upper bound on the stratification of the mean state. First of all, we want to prove with a scaling analysis that in our case, the Rossby number, $R_{0}$, is of $\mathrm{O}(1)$. The equatorial Rossby number is defined as $R_{0}=\frac{U}{\beta y L}$. If we assume $y$ to be the equatorial radius of deformation $(\simeq 200 \mathrm{~km})$ and $L$ the meridional length scale of the disturbances $\left(\simeq 2^{\circ}\right)$, for a mean flow with $U_{0}=100 \mathrm{~cm} / \mathrm{s}$, then $R_{0}=0.99$. Therefore, the critical condition imposed on the mean stratification in order to have baroclinic instability is $R_{i} \leq 1$, i.e.

$N^{2} \leq \frac{U^{2}}{H^{2}}$.

For our simplified system, $N^{2}=g \alpha \frac{\partial T}{\partial z}$, where we have combined the equation of state (1) and the definition of BruntVäisälä frequency $N^{2}=-\frac{g}{\rho_{0}} \frac{\partial \rho}{\partial z}$. Consequently, Eq. (15) becomes

$\frac{\partial T}{\partial z} \leq \frac{U^{2}}{H^{2} g \alpha}$.

Using the height of the mean flow vertical variation $\mathrm{H}=150 \mathrm{~m}$ and the value of $\alpha$ adopted in this study (see Sect. 2), we obtain that for baroclinic instability to be possible near the equator, the critical temperature change over a $90 \mathrm{~m}$ deep frontal layer is

$\nabla_{z} T=0.95^{\circ} \mathrm{C}$.

In EXP 4 , we used $\nabla_{z} T=0.9^{\circ} \mathrm{C}$ and we showed that the instability is very weak, while in EXP 5 we used $\nabla_{z} T=$ $0.45^{\circ} \mathrm{C}$ and the amplitude of the instabilities is larger. In summary, we can conclude that the critical $\nabla_{z} T$ required for baroclinic instability is of $\mathrm{O}(1)^{\circ} \mathrm{C}$ for a $90 \mathrm{~m}$ deep frontal layer.

Orlanski (1986) showed that an environment with weak static stability in the lower levels of the atmosphere can support shallow, very unstable mesoscale baroclinic waves. These results were confirmed by Nakamura (1988) who concluded that a reduced static stability generally shortens the horizontal scale and enhances the growth rate of the unstable waves. Both these results have been obtained for Rossby numbers of $\mathrm{O}(1)$.

\subsection{Baroclinic instability near the equator}

A growing baroclinic disturbance draws its energy from the eddy potential energy (Orlanski and Cox, 1973). Therefore, if we want to demonstrate that the oscillations grown in the numerical simulations described in Sect. 3.1 have a baroclinic nature, then we have to show the energetics involved in the generation of the instabilities. If we define a mean quantity as

$G(y, z, t)=\frac{1}{L} \int_{x_{0}}^{x_{0}+L} g(x, y, z, t) d x$,

where $L$ is the length of the channel, then the perturbation quantities are

$g^{\prime}(x, y, z, t)=g(x, y, z, t)-G(y, z, t)$. 
If we apply the same averaging procedure to the equations of motions, as described in Orlanski and Cox (1973), the time rate of change of eddy kinetic energy can be obtained (see their Eq. 5.19) and defined as

$$
\begin{aligned}
& \int_{0}^{D} \int_{H}^{0} K_{e t} d z d y=-\iint \overline{u^{\prime} v^{\prime}} \bar{U}_{y} d z d y \\
& -\iint\left(\overline{u^{\prime} w^{\prime}} \bar{U}_{z}+\overline{v^{\prime} v^{\prime}} \bar{V}_{y}+\overline{v^{\prime} w^{\prime}} \bar{V}_{z}\right) d z d y \\
& +\iint \rho_{0} g \alpha \overline{T^{\prime} w^{\prime}} d z d y+\text { Dissipation, }
\end{aligned}
$$

where the bar indicated a zonal average, $D$ is the latitudinal extension of the domain and the other variables have been already defined. Since the initial mean flow is characterized by a velocity $\bar{U}(y, z)$ and the fluctuation vertical velocity is usually smaller than the horizontal velocity, we have split the Reynolds stresses into two terms. The first term, on the righthand side of Eq. (20), is the dominant Reynold stress. The first two integral terms on the right hand side of Eq. (20) represent the conversion of mean kinetic energy to eddy kinetic energy through Reynolds stresses. The conversion of eddy potential energy into eddy kinetic energy is given by the third term on the right-hand side of Eq. (20) and is usually called the baroclinic conversion term. The last term represents the loss of energy through dissipation. If we do not integrate in $y$ and $z$ other terms due to advection and pressure, their work has to be taken into consideration (see Masina et al. (1999) for details). Since we are interested in explaining how the waves are generated, we can focus our attention only on the first and the third term on the right-hand side of Eq. (20), since they represent the sources of eddy kinetic energy.

Figures $3 \mathrm{a}$ and $3 \mathrm{~b}$ show the quantity $\overline{T^{\prime} w^{\prime}}$ averaged in time for EXP 2 and EXP 3, respectively. In EXP 3, the baroclinic conversion term is one order of magnitude larger than in EXP 2, confirming that the magnitude of baroclinic conversions during wave growth increases with an increase in depth of the frontal layer. Since $T^{\prime}$ is comparable in the two experiments, it is the vertical velocity that is one order of magnitude larger in EXP 3.

The comparison between Fig. 3a and Fig. 3b also shows that the vertical scale of the unstable waves is determined by the depth of the frontal layer. The meridional scale and zonal wavelength of the unstable oscillations are the same in both cases and seem not to be affected by the frontal layer depth.

The barotropic conversion term $-\overline{u^{\prime} v^{\prime}} \bar{U}_{y}$ averaged in time (not shown) is one order of magnitude smaller than the baroclinic conversion term in both the experiments and is everywhere negative with the exception of two shallow positive surface regions (above $20 \mathrm{~m}$ ) between $2^{\circ} \mathrm{S}$ and $2^{\circ} \mathrm{N}$. This means that the barotropic conversion is negligible with respect to the baroclinic conversion and that tends to suppress the instability mechanism, since it mainly transforms eddy kinetic energy to mean flow kinetic energy.

The meridional heat flux $\left\langle\overline{T^{\prime} v^{\prime}}\right\rangle$ (where the brackets denote a time mean) associated with the instability waves is shown in Fig. 4, together with the zonal mean temperature field for
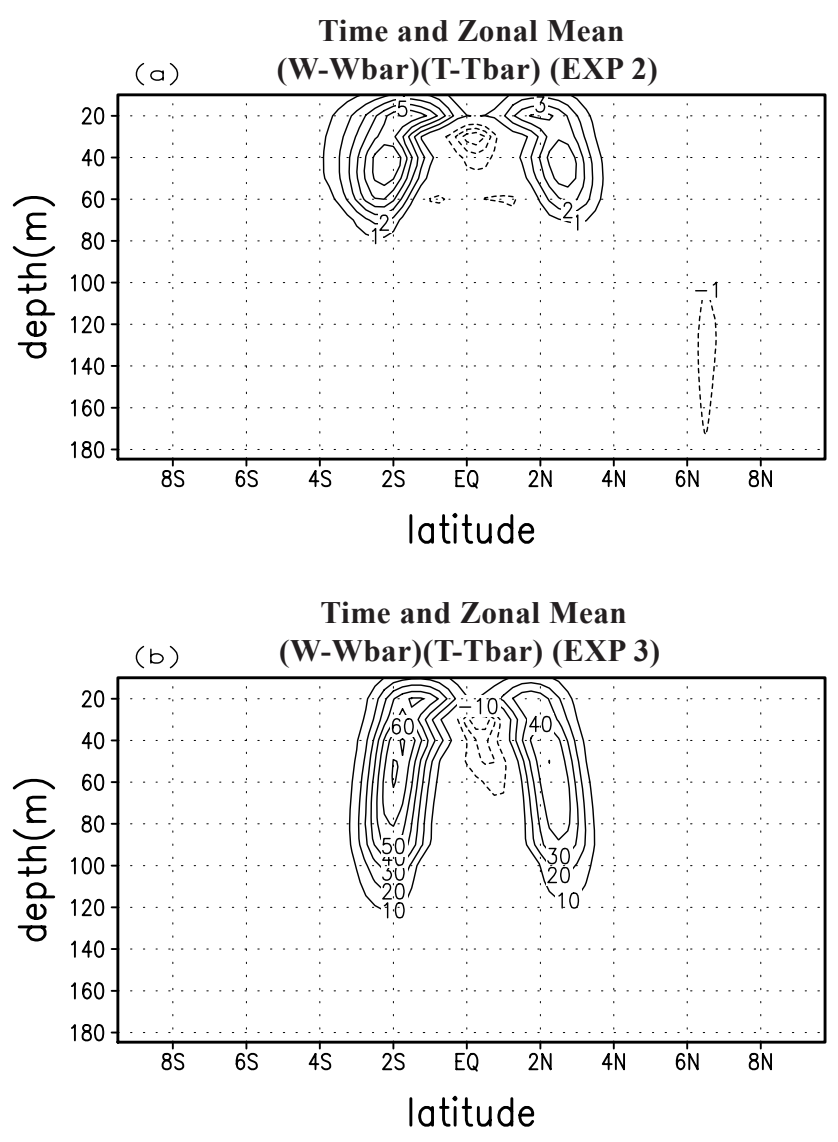

Fig. 3. Depth-latitude sections of temporally and zonally averaged $w^{\prime} T^{\prime}$ for: (a) EXP 2 and (b) EXP 3. Values are multiplied by $10^{6}$ in both panels and the contour intervals are $1 \mathrm{~cm}^{\circ} \mathrm{C} / \mathrm{s}$ in (a) and $10 \mathrm{~cm}^{\circ} \mathrm{C} / \mathrm{s}$ in (b).

EXP 3. In the equatorial region, the mean meridional temperature gradient is opposite with respect to mid-latitudes due to the upwelling at the equator. The heat flux of a baroclinic wave at mid-latitudes in the Northern Hemisphere is positive and the release of available potential energy to the growing waves is associated with cold fluid moving equatorward and warm fluid moving poleward. In our case, $\overline{T^{\prime} v^{\prime}}$ is negative north of the equator, but is still down the mean temperature gradient, as it is at mid-latitudes and therefore, it is still associated with a release of available potential energy and tends to warm up the cold surface water near the equator. The vertical slope of the isotherms, with respect to the initial condition in the frontal layer (see Fig. 1c), is consistent with the release of available potential energy.

In conclusion, both the meridional and vertical heat fluxes are consistent with the interpretation of these instabilities as baroclinic instability waves whose primary source of energy is the available potential energy and not the kinetic energy of the mean flow. Indeed, the barotropic conversion term is consistent with an increasing of the mean kinetic energy. This result is in agreement with the conclusions by Nakamura (1993a, 1993b) that a weak barotropic shear tends to shape baroclinic instability in such a way as to produce a counter- 
Tbar at $\mathrm{t}=65$ (shaded) and Time and Zonal Mean Heat Flux (EXP 3)

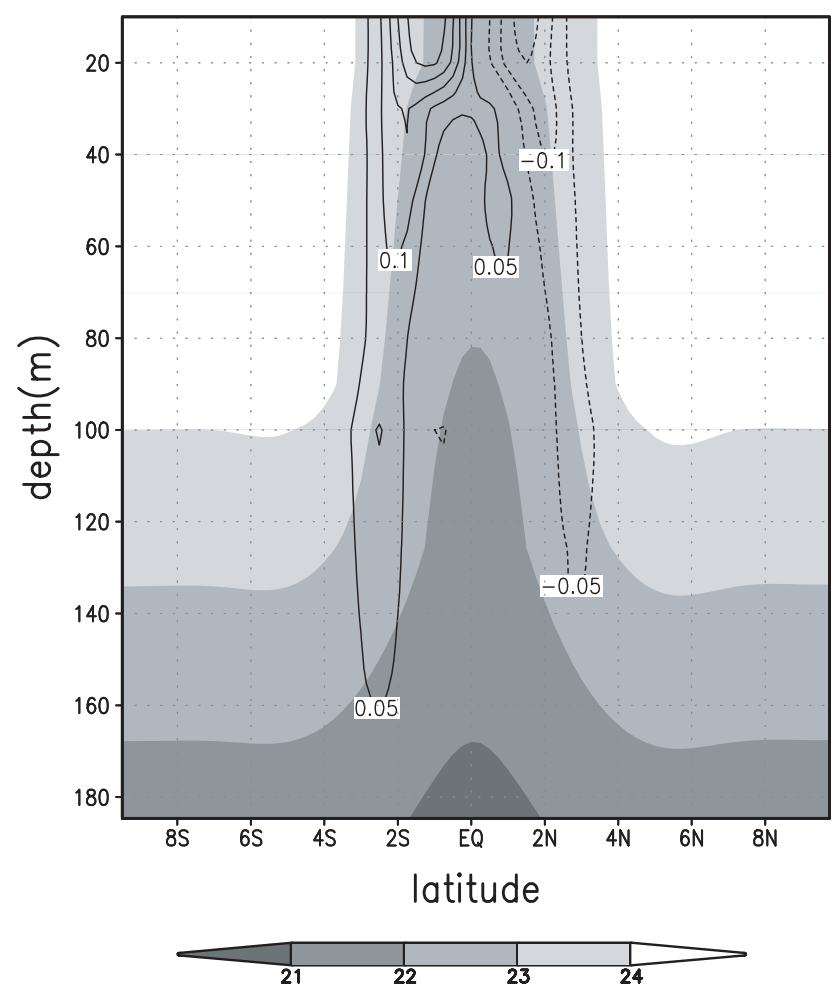

Fig. 4. Depth-latitude section of zonally averaged temperature (shaded, c.i. $=1^{\circ} \mathrm{C}$ ) after 65 days of integration of EXP 3 and time and zonal mean $\mathrm{v}^{\prime} \mathrm{T}^{\prime}\left(\mathrm{c} . \mathrm{i} .=0.05 \mathrm{~cm}{ }^{\circ} \mathrm{C} / \mathrm{s}\right)$.

gradient momentum flux that reinforces the barotropic shear. This positive feedback, also known as the "barotropic governor", prevents the eddies from using all the available potential energy stored in the domain and suggests the natural tendency of baroclinic waves to decay barotropically.

These experiments confirm that baroclinic instability is likely to occur in the equatorial region if upwelling at the equator establishes a meridional temperature gradient that supplies potential energy available to be used in growing the instability, as in the traditional mid-latitudes baroclinic instability mechanism. McCreary and Yu (1992) showed that in a 2.5-layer model the equatorial temperature front is important in generating instabilities with characteristics similar to TIWs. In their study, the instability involves conversion of mean potential energy associated with the variable upperlayer temperature front to eddy potential energy, whereas traditional baroclinic instability is an energy sink. Nevertheless we believe that this new type of "frontal instability" and the baroclinic instability mechanism found in our study are related. The fact that in the study of McCreary and Yu (1992) the traditional baroclinic instability is not an energy source for the oscillations might be due to the inadequacy of a 2.5layer model in reproducing correctly the baroclinic instability mechanism in the equatorial region. Ripa's $(1983,1991)$ results support this conclusion, since he found that stabil- ity conditions for ageostrophic flows at the equator are more easily violated in a multi-layer model as the number of layers increases.

\subsection{EXP 6, EXP 7, EXP 8: initial conditions and results}

We perform a series of three experiments in order to explore how different mean flow conditions (in particular, different vertical shears in the mean flow) affect the generation and propagation of unstable waves. Three initial mean flow conditions that have the same meridional and vertical structure but different maximum velocity are integrated in time in the channel domain; these jets are westward and are symmetric about the equator. As listed in Table $1, U_{0}=50 \mathrm{~cm} / \mathrm{s}$ in EXP 6 , $U_{0}=100 \mathrm{~cm} / \mathrm{s}$ in EXP 7 and $U_{0}=200 \mathrm{~cm} / \mathrm{s}$ in EXP 8. The $y$ and $z$ dependence of the mean flow is given by Eq. (8). The temperature field is obtained as in Eq. (12), using the same $T_{0}(z)$ and applying a $90 \mathrm{~m}$ deep frontal layer to all the experiments. The model configuration that we use differs from that described and adopted in the previous series of experiments in only one aspect: the zonal length of the domain $L$ has been increased from $10^{\circ}$ to $15^{\circ}$. This change allows for waves with a greater wavelength to grow. We increase $L$, since longer waves are expected to grow in a mean flow with a vertical shear stronger (EXP 8) than that used in the first set of experiments (Sect. 3.1).

EXP 6 is stable. As in EXP 2, some wave structures develop early during the integration, but they never grow in the sense that the eddy kinetic energy does not grow exponentially in time as it should during an instability process. The oscillations saturate and then dissipate on a time scale of 2 months.

EXP 7 shows that two zonal wavelengths are contained in the length of the domain $L$, while in EXP 8 the dominant wavelength of the unstable waves is $L$. This means that the wavelengths are $\simeq 825 \mathrm{~km}$ and $1650 \mathrm{~km}$ in EXP 7 and EXP 8 , respectively.

The experiment with the maximum vertical shear (EXP 8) is also the most unstable, with a wave amplitude that is one order of magnitude greater than that obtained in EXP 7. The two cases are compared in Fig. 5a and b, which show the perturbation meridional velocity as a function of latitude and time. From the figures we can clearly see the difference in magnitude, as well as in period. In EXP 7, the period is approximately 20 days, while it increases to 30 days in EXP 8 . Since we also know the wavelength of the fastest-growing wave in each case, we can estimate the phase speeds of the two waves as

$$
\begin{aligned}
& \operatorname{EXP} 7: c \simeq \frac{L / 2}{20 \text { days }}=\frac{7.5^{\circ}}{20 \text { days }} \simeq 43 \mathrm{~cm} / \mathrm{s}, \\
& \operatorname{EXP~} 8: c \simeq \frac{L}{30 \text { days }}=\frac{15^{\circ}}{30 \text { days }} \simeq 58 \mathrm{~cm} / \mathrm{s} .
\end{aligned}
$$

The first conclusion that we can make from these experiments is that in the equatorial region of a continuously stratified fluid there is a critical vertical shear for baroclinic instability. A second conclusion is that the dependence of the 

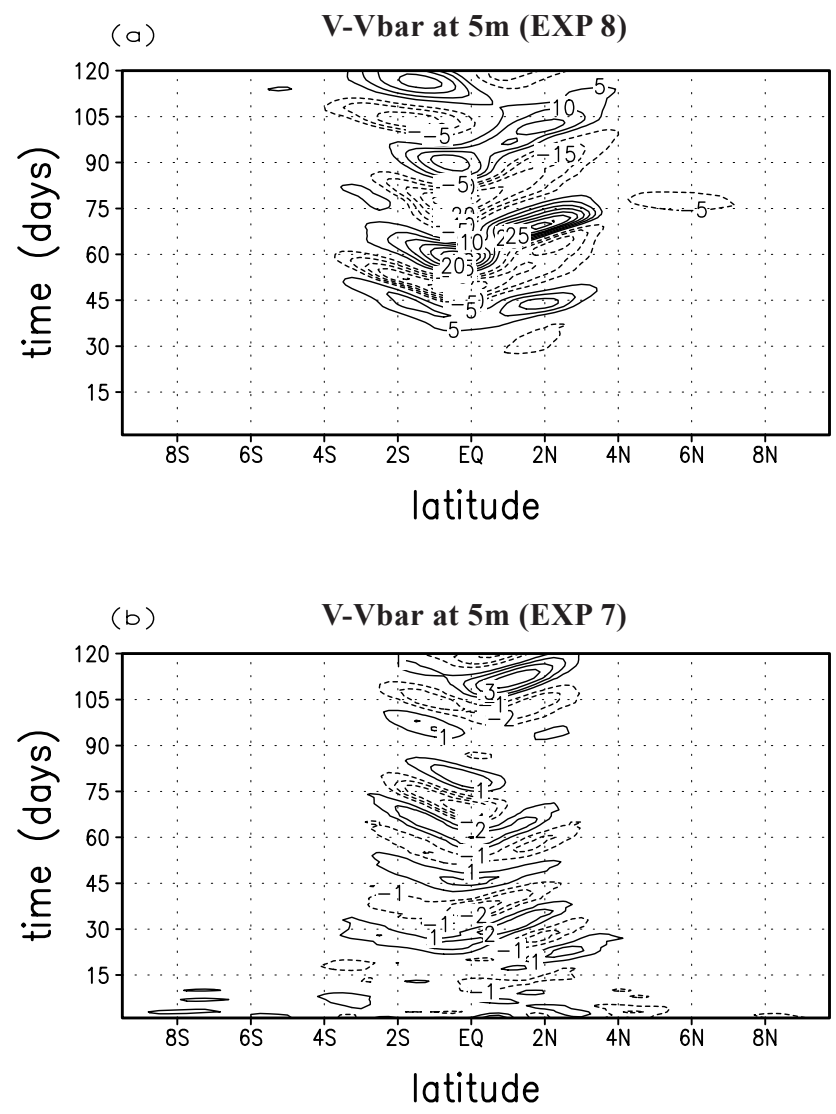

Fig. 5. Time-latitude diagrams of perturbation meridional velocity at $5 \mathrm{~m}$ for: (a) EXP 8 and (b) EXP 7. Contour interval is $5 \mathrm{~cm} / \mathrm{s}$ in (a) and $1 \mathrm{~cm} / \mathrm{s}$ in (b).

wavelengths obtained in EXP 7 and EXP 8 on the length of the domain $L$ is in agreement with the results obtained by Charney (1947) who showed that the wavelength of the fastest-growing wave is directly proportional to the vertical shear of the mean flow. Of course we cannot directly apply the results of the Charney model to our case, because they were derived under the constraints of quasi-geostrophic theory, but it is significant that the wave characteristics in the equatorial region show a similar dependence on mean flow conditions. Nakamura (1988) showed that when nongeostrophic effects become important the scale of the most unstable Eady's mode becomes sensitive to changes in the mean flow velocity shear. Under nongeostrophic condition, he showed that the horizontal scale of the disturbance is the modified Rossby radius of deformation

$L_{R} \simeq \frac{H\left(N^{2}+\Lambda^{2}\right)^{\frac{1}{2}}}{f}$,

where $\Lambda$ is the vertical shear. This conclusion is in agreement with the results of our experiments.

The estimated values of the phase speeds show that the phase velocity of the unstable waves becomes approximately $30 \%$ faster when the maximum mean flow velocity and the mean flow vertical shear is doubled. These results are also consistent with the conclusions of Seigel (1985) who showed that changes in the period of instabilities generated in the SEC are due to variations in the intensity of the current itself and in its vertical shear. Seigel (1985) found that the phase speed of the most unstable wave increases as the intensity of the westward current is doubled.

\section{Mean flow II: a surface westward jet asymmetric about the equator}

The second mean flow that we use to run another numerical experiment is a surface westward jet asymmetric about the equator. In particular, the axis of symmetry of the jet is moved from the equator (Sect. 3) to $2^{\circ} \mathrm{N}$, in order to better simulate the SEC in the eastern Pacific. However, the absence of the EUC is crucial in making this idealistic case different from the realistic equatorial zonal circulation.

\subsection{EXP 9: initial condition and results}

For this experiment (EXP 9), we use a mean flow $U(\theta, z)$ with the same latitudinal and vertical structures as the one used in the first set of experiments and with $U_{0}=100 \mathrm{~cm} / \mathrm{s}$. In this case, however, the jet is centered at $2^{\circ} \mathrm{N}$ and is confined to a latitudinal region between $2^{\circ} \mathrm{S}$ and $6^{\circ} \mathrm{N}$. The zonal length of the domain is $10^{\circ}$ of longitude. The temperature field $T(y, z)$ has been calculated geostrophically from $U(\theta, z)$, as in Eq. (12). No frontal layer is imposed on the flow. The zonal mean flow and temperature fields at $\mathrm{t}=0$ are shown in Fig. 6.

Since the zonal flow is not centered on the equator, the temperature field is strongly asymmetric because, even for the same $\frac{\partial U}{\partial z}$, the meridional temperature gradient $\frac{\partial T}{\partial y}$ is different at different latitudes due to the $y$ dependence of the Coriolis parameter. The geostrophically balanced temperature profile shown in Fig. 6 is particularly interesting because it is characterized by a strong vertical shear but almost no latitudinal shear south of $2^{\circ} \mathrm{N}$. Conversely, there is strong latitudinal shear and weak vertical shear north of $2^{\circ} \mathrm{N}$. This implies that the source of eddy available potential energy $\left(\propto \frac{\nabla_{h} T}{T_{z}}\right)$ is mostly concentrated in the northern half of the jet. Unstable oscillations develop both north and south of the equator (not shown), but the northern waves are clearly visible in the temperature field, while the southern instabilities can be detected only in the zonal velocity field due to the absence of a temperature front south of the equator. However, the zonal velocity has a stronger meridional shear south of the equator than north of it. This asymmetry is clearly an indication of two different instability processes occurring at different latitudes. The perturbation meridional velocity as a function of time and latitude $\left(5^{\circ} \mathrm{S}-5^{\circ} \mathrm{N}\right)$ at the first model level (EXP 9) is shown in Fig. 7. The oscillations north and south of the equator grow and decay twice during the 8 month numerical integration.

The horizontal structure of the two waves is similar, as is evident from Fig. 8, in which a horizontal section of tem- 


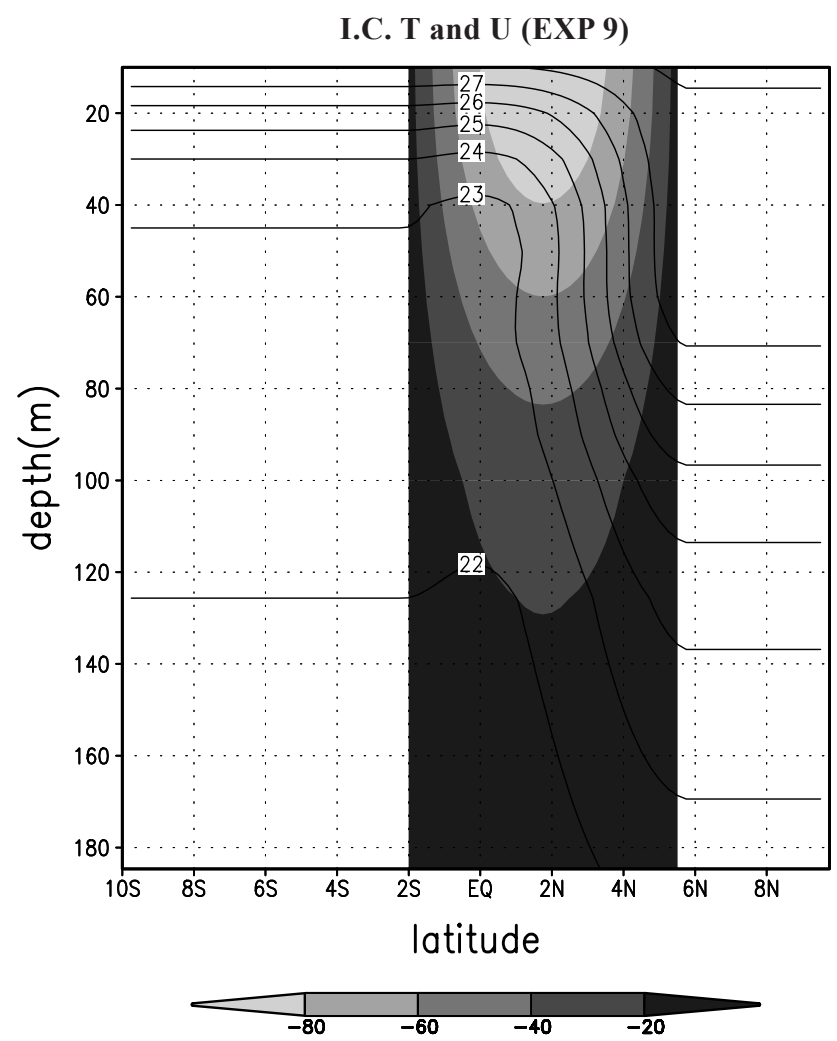

Fig. 6. Depth-latitude section of zonally uniform horizontal velocity (shaded) and temperature field (contour) used as the initial condition for EXP 9. Contour intervals are $1^{\circ} \mathrm{C}$ for temperature and $20 \mathrm{~cm} / \mathrm{s}$ for velocity.

perature and perturbation velocity vectors is shown at the channel surface. The snapshot, taken when both the waves are present, shows that the northern wave is centered on the strong temperature front while the southern wave straddles the $2^{\circ} \mathrm{S}$ latitude that corresponds to the southern boundary of the westward jet. The temperature front, near $4^{\circ} \mathrm{N}$ presents a barrier to the waves and prevents them from extending further north.

\subsection{Barotropic and baroclinic instability waves}

In order to determine the nature of the two different kinds of instability which grow in EXP 9, we have to show the energetics involved in the generation of the two unstable modes. In Figs. 9a and 9b, we show the barotropic and baroclinic conversion term, respectively (first and third term on the right hand side of Eq. 20), as a function of time and latitude between $5^{\circ} \mathrm{S}$ and $5^{\circ} \mathrm{N}$. Both terms are integrated vertically in the upper 12 model levels to which the instabilities are confined and zonally over the domain length.

The baroclinic conversion term is different from zero only north of $2^{\circ} \mathrm{N}$ when the northern instability is present. The barotropic term shown in Fig. 9a is positive in two regions. The first region is between $1^{\circ} \mathrm{S}$ and $2^{\circ} \mathrm{N}$ in latitude and between the second and fourth month of integration. The sec-

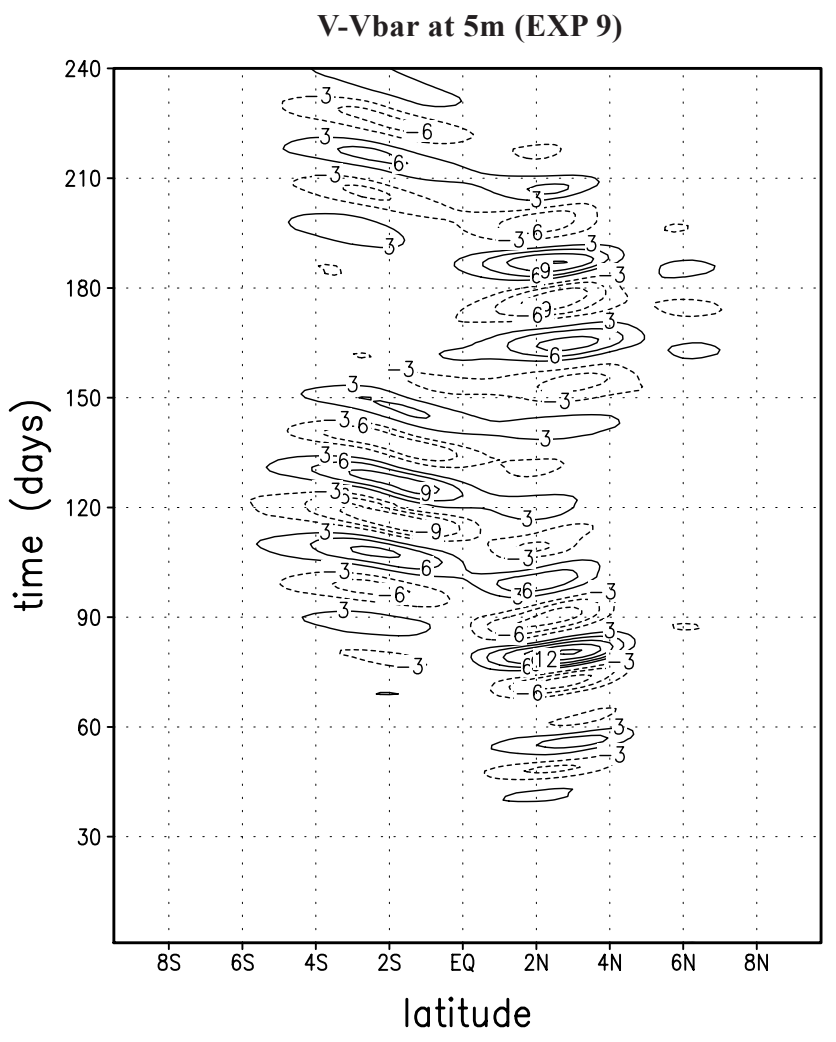

Fig. 7. Time-latitude diagram of perturbation meridional velocity at $5 \mathrm{~m}$ for EXP 9. Contour interval is $3 \mathrm{~cm} / \mathrm{s}$.

ond region is smaller and weaker, and occurs during the seventh month of integration between the equator and $1^{\circ} \mathrm{S}$.

In these regions, mean kinetic energy of the system is converted to eddy kinetic energy, while in the regions of negative barotropic conversion an inverse barotropic cascade is occurring, indicating that eddy kinetic energy is converted to mean kinetic energy. This last process suppresses the generation of barotropic instabilities. We can now follow the generation and dissipation of eddy kinetic energy (not shown) in terms of the barotropic and baroclinic conversions. The northern instability is first generated after two months of integration through a baroclinic instability process. Then the eddy kinetic energy is dissipated through an inverse barotropic cascade (Nakamura, 1988) that alters the mean flow in such a way as to trigger the generation and amplification of a barotropic mode south of the equator. This mode then decays and converts eddy kinetic energy into mean kinetic energy such that the mean flow configuration, in turn excites a new baroclinic mode north of $2^{\circ} \mathrm{N}$ and the cycle repeats itself.

\section{Discussion and conclusions}

The equatorial region represents a unique oceanic system due to the dynamical situation that arises from the combined effects of wind forcing and of vanishing rotation. Geostrophically, for a given vertical shear in velocity, the horizontal 


\section{$T$ and Velocity Vectors at $t=110,5 m($ EXP 9)}

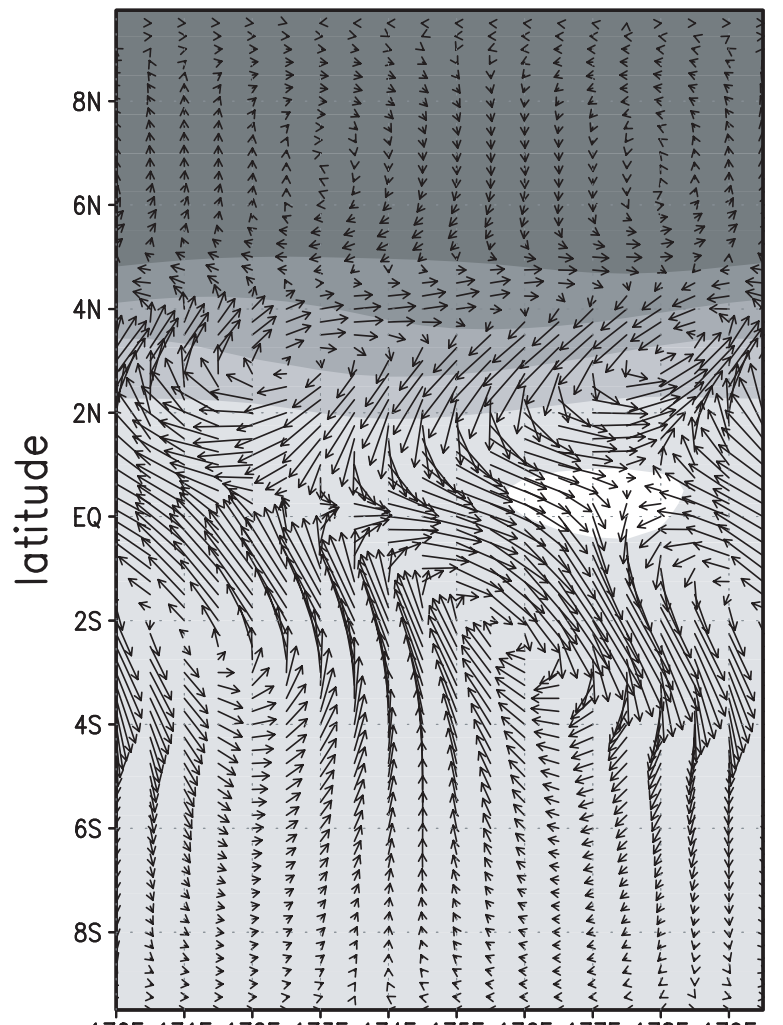

170E 171E 172E 173E 174E 175E 176E 177E 178E 179E

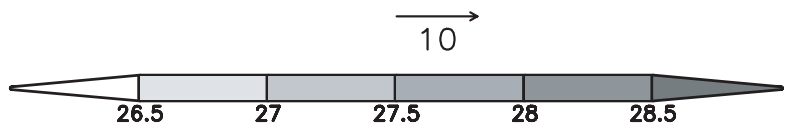

Fig. 8. Horizontal snapshot of temperature (shaded) and perturbation velocity vectors at $5 \mathrm{~m}$ after 110 days of integration of EXP 9 . Contour interval is $0.5^{\circ} \mathrm{C}$ for temperature and the unit vector is $10 \mathrm{~cm} / \mathrm{s}$.

temperature gradient is proportional to the Coriolis parameter $f$. There is a consequent flattening of the isotherms as the equator is approached. However, surface winds, that are mainly easterly in the equatorial region, induces an Ekman drift which is to the right of the wind in the Northern Hemisphere and to the left in the Southern Hemisphere. The consequent Ekman divergence forces cold water to upwell along the equator with a consequent outcropping of isotherms at the surface. This allows for the possibility of extraordinarily strong temperature fronts in the equatorial region that can supply potential energy available to be used in growing the instability, as in the traditional mid-latitudes baroclinic instability mechanism. In summary, the potential for baroclinic instability near the equator seems to be as large as at midlatitudes. In addition to this, the particularly strong horizontal shears that characterize the equatorial zonal currents also allow for the possibility of barotropic instability.

A three-dimensional channel model of the continuously stratified equatorial region has been integrated in time using (a) Barotropic Conversion (1-180m) (EXP 9)

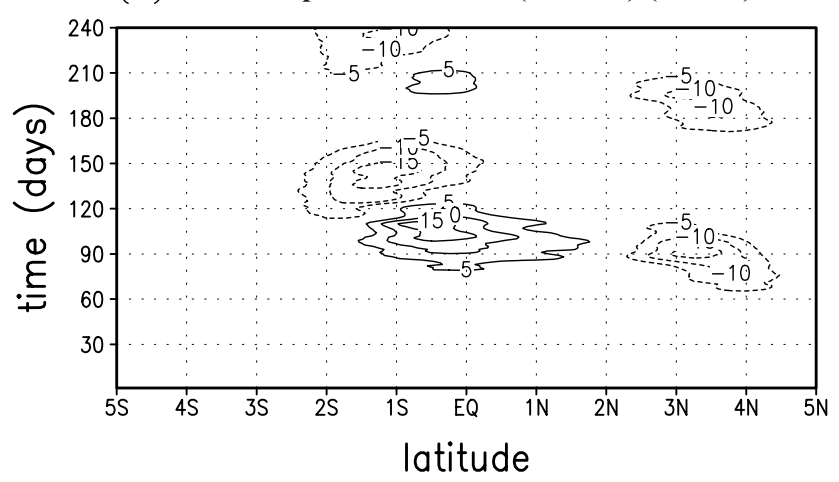

(b) Baroclinic Conversion (1-180m) (EXP 9)

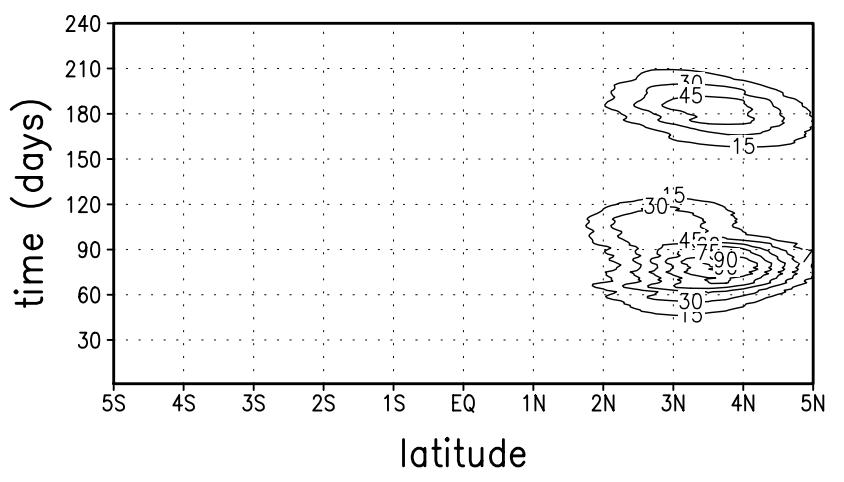

Fig. 9. Time-latitude diagrams of vertically (in the upper $180 \mathrm{~m}$ ) and zonally averaged barotropic conversion (a) and baroclinic conversion (b) for EXP 9. Values are multiplied by $10^{6}$ in both panels and the contour intervals are $5 \mathrm{~cm}^{2} / \mathrm{s}^{3}$ in (a) and $15 \mathrm{~cm}^{2} / \mathrm{s}^{3}$ in (b).

different mean flow configurations, in order to examine the stability of equatorial zonal currents. Surface westward jets, either symmetric about the equator or confined to the Northern Hemisphere, are explored to simulate the stability of the SEC. Surface westward jets symmetric about the equator are unstable if the maximum velocity of the jet is $\geq 100 \mathrm{~cm} / \mathrm{s}$ and if a frontal layer $(\geq 90 \mathrm{~m})$ is introduced. In fact, the presence of a frontal layer where $N^{2}=0$ or $\simeq 0$ seems to favour the generation of unstable waves a few degrees $\left(2^{\circ} \mathrm{S}-2^{\circ} \mathrm{N}\right)$ from the equator. Moreover, we have demonstrated that the depth of the frontal layer is a crucial parameter for the generation of unstable waves. A $60 \mathrm{~m}$ deep frontal layer is not sufficient to destabilize a westward jet with a maximum velocity of $100 \mathrm{~cm} / \mathrm{s}$. If the frontal layer is increased to $90 \mathrm{~m}$, the same jet becomes unstable. A critical value for the vertical mean stratification has been estimated in the nongeostrophic regime. It has been found that a critical $\nabla T$ of $\mathrm{O}(1)^{\circ} \mathrm{C}$ for a $90 \mathrm{~m}$ deep frontal layer is required for baroclinic instability to occur. These results are in agreement with previous findings (Philander, 1976 and Cox, 1980) that an increasing divergence induced by a reduction of the static stability tends to destabilize westward currents. Moreover, our results suggest that Stone's $(1966,1970)$ results about ageostrophic $\left(R_{i}, \leq 1\right)$ baroclinic instability are also valid in the equatorial 
region.

The westward propagating unstable waves that arise in the channel model in westward surface jets with a maximum velocity of $100 \mathrm{~cm} / \mathrm{s}$ (EXP 7) have a wavelength and period that are in good agreement with the oscillations excited in the eastern Pacific Ocean at, and just north, of the equator (Qiao and Weisberg, 1995; Halpern et al., 1988). However, there is observational evidence of oscillations also further north of the equator. Altimeter data (Perigaud, 1990) show westward propagating eddies along $5^{\circ} \mathrm{N}$, and current meter and thermistor chain moorings reveal monthly period oscillations in velocity and temperature in the vicinity of the NECC, along $7^{\circ} \mathrm{N}$ (McPhaden, 1996). Analysis of current and density fields (Luther and Johnson, 1990) shows the existence of multiple instabilities: one close to the equator, another at the equatorial front from $3^{\circ} \mathrm{N}$ to $6^{\circ} \mathrm{N}$ and one in the thermocline of the NECC. The results of this study obtained for a surface westward jet asymmetric about the equator (EXP 9) and resembling the SEC in the eastern Pacific have a "two-modes" structure, which might be related to the multiple oscillations observed at different latitudes. In fact, in the case of a westward jet that is asymmetric about the equator, we have shown evidence of two kinds of unstable waves: one at the southern edge of the westward jet and the other at the temperature front between $2^{\circ} \mathrm{N}$ and $4^{\circ} \mathrm{N}$. In both our idealized experiments and observations the waves are confined vertically in the frontal layer.

Furthermore, we have confirmed Eady's and Charney's results concerning the dependence of the maximum growth rate and zonal wavelength of the fastest-growing wave on the vertical shear of the jet. In fact, it has been shown that even when the quasi-geostrophic theory is not applicable the growth rate, the zonal wavelength and the phase speed of the unstable waves is directly proportional to the mean current vertical shear.

The eddy fluxes and energy analysis of the experiments done with the westward jet symmetric about the equator indicate that the equatorial waves grown in the channel model draw their eddy kinetic energy from the available potential energy via the baroclinic conversion term. This result is consistent with the conclusions of an energy analysis performed to understand the nature of instabilities simulated in a numerical model and with characteristics similar to TIWs (Masina et al., 1999). Those conclusions indicated baroclinic instability at the northern equatorial temperature front as the triggering mechanism which developed the oscillations. Also, McCreary and $\mathrm{Yu}$ (1992) showed that the equatorial temperature front is important in generating TIWs. In their study, the instability, which involves conversion of mean potential energy associated with the variable upper-layer temperature front to eddy potential energy, is named frontal instability.

In the experiment performed with a surface westward jet asymmetric about the equator (EXP 9), the oscillations generated north of the equator have a baroclinic nature, while those generated south of the equator draw their energy from the horizontal shear of the mean flow via barotropic instability. The latter waves are generated from the inverse barotropic cascade that inhibits and stops the baroclinic evolution of nearby waves; the cascade alters the mean flow in such a way as to trigger the amplification of a barotropic mode. The double energy nature of the oscillations in these simplified experiments might be related to both observational and other modelling studies. An energy analysis of measurements (Luther and Johnson, 1990) shows a large mean flow to eddy kinetic energy conversion just north of the equator and a large mean flow to eddy potential energy conversion at the equatorial front between $3^{\circ} \mathrm{N}$ and $6^{\circ} \mathrm{N}$. In a continuously stratified $\beta$-plane model (Proehl, 1998), it is found that barotropic instability dominates in the region between the Equatorial Undercurent and the northern branch of the SEC. In Proehl's (1998) study, the role of the strong temperature front is not investigated, but it is suggested that it might play a role and introduce baroclinic instability when more realistic vertical stratification is introduced. A 2- $\frac{1}{2}$ layer model forced with climatological winds generates two westward-propagating waves (Donohue and Wimbush, 1998). An eddy-energy budget provides evidence that the two-wave processes are generated at different source regions. One energy source is located near the equator along the equatorward edge of the SEC and is dominated by upperlayer barotropic conversion. The second one, between $2^{\circ}$ and $5^{\circ}$, is a combination of upper-layer barotropic conversion along the poleward edge of the SEC and baroclinic conversion near the core of the SEC. Finally, in a numerical model forced with realistic winds, Masina et al. (1999) confirmed that the instabilities generated in the eastern Pacific consist of two components phase locked to each other. One of the two components develops between $2^{\circ} \mathrm{S}$ and the equator, and barotropic instability plays a significant role in its generation.

In conclusion, surface westward equatorial jets with zonal velocity of $\mathrm{O}(1) \mathrm{m} / \mathrm{s}$ can be unstable and sustain growing instabilities in the frontal layer via baroclinic and barotropic instability processes. The direction and the typical values of the phase speed obtained in the case of a westward jet are in good agreement with the properties of the wind-driven unstable waves observed in the equatorial Pacific (TIWs).

Acknowledgements. The author is indebted to S. G. Philander for his assistance throughout this study which was mostly carried out at Princeton University. I thank A. B. G. Bush for reading an earlier version of the manuscript and making many helpful comments.

The Editor in Chief thanks J. Vialard and another referee for their help in evaluating this paper.

\section{References}

Benilov, E. S. and Sakov, P. V.: On the linear approximation of velocity and density profiles in the problem of baroclinic instability, J. Phys. Ocean., 29, 1374-1381, 1999.

Chang, P. and Philander, S. G. H.: Rossby Wave packets in baroclinic mean currents, Deep-Sea Res., 36, 17-37, 1989.

Charney, J. G.: The dynamics of long waves in a baroclinic westerly current, J. Meteor., 4, 135-162, 1947. 
Cox, M. D.: Generation and Propagation of 30-day Waves in a Numerical Model of the Pacific, J. Phys. Ocean., 10, 1168-1186, 1980.

Donohue, K.A. and Wimbush. M.: Model results of flow instabilities in the tropical Pacific Ocean, J. Geophys. Res., 103, 21401 $21412,1998$.

Eady, E. T.: Long waves and cyclone waves, Tellus, 1, 33-52, 1949.

Gill, A.: Atmospheric-Ocean Dynamics, International Geophysical Series No. 30. London: Academic Press, 662, 1982.

Halpern, D., Knox, R. A., and Luther, D. S.: Observations of 20-day period meridional current oscillations in the upper ocean along the Pacific equator, J. Phys. Ocean., 18, 1514-1534, 1988.

Kennan, S. C. and Flament, P. J.: Observations of a Tropical Instability Vortex, J. Phys. Ocean., 30, 2277-2301, 2000.

Kuo, H. L.: Dynamic instability of two-dimensional non-divergent flow in a barotropic atmosphere, J. Meteorol, 6, 105-122, 1949.

Legeckis, R.: Long waves in the eastern equatorial Pacific Ocean: A view from a geostationary satellite, Science, 197, 1179-1181, 1977.

Lipps, F. B.: Stability of jets in a divergent barotropic fluid, J. Atmos. Sci., 20, 120-129, 1963.

Luther, D. S., Knox, R. A., and Halpern, D.: Eddy heat fluxes in the upper equatorial Pacific Ocean along $140^{\circ} \mathrm{W}$, EOS, 68, 1321, 1987.

Luther, D. S. and Johnson, E. S.: Eddy energetics in the upper equatorial Pacific during the Hawaii-to-Tahiti Shuttle Experiment, J. Phys. Ocean., 20, 913-944, 1990.

Masina, S. and Philander, S. G.: An analysis of Tropical Instability Waves in a numerical model of the Pacific Ocean. Part I: Spatial variability of the waves, J. Geophys. Res., 104, C12, 29613 29636, 1999.

Masina, S., Philander, S. G., and Bush, A. B. G.: An analysis of Tropical Instability Waves in a numerical model of the Pacific Ocean. Part II: Generation and energetics of the waves, J. Geophys. Res., 104, C12, $29637-29661,1999$.

McCreary Jr, J. P. and Yu, Z.: Equatorial dynamics in a $2 \frac{1}{2}$-layer model, Prog. Oceanogr., 29, 61-132, 1992.

McPhaden, M. J.: Monthly period oscillations in the Pacific North Equatorial Countercurrent, J. Geophys. Res., 101, 6337-6359, 1996.

McPhaden, M. J. and Ripa, P.: Wave-mean flow interactions in the equatorial ocean, Annu. Rev. Fluid Mech., 22, 167-205, 1990.

Nakamura, N.: Scale selection of baroclinic instability-Effects of stratification and nongeostrophy, J. Atmos. Sci., 45, 3253-3267, 1988.

Nakamura, N.: An illustrative model of instabilities in meridionally and vertically sheared flows, J. Atmos. Sci., 50, 357-375, 1993a.

Nakamura, N.: Momentum flux, flow symmetry, and the nonlinear barotropic governor, J. Atmos. Sci., 50, 2159-2179, $1993 \mathrm{~b}$.

Orlanski, I.: Localized baroclinicity: A source for meso- $\alpha$ cyclones, J. Atmos. Sci., 43, 2857-2885, 1986.

Orlanski, I.: and Cox, M. D.: Baroclinic instability in ocean currents, Geophys. Fluid Dynam., 4, 297-332, 1973.

Pacanowski, R. C. and Philander, S. G. H.: Parameterization of vertical mixing in numerical models of Tropical Oceans. J. Phys. Ocean., 11, 1443-1451, 1981.

Pacanowski, R., Dixon, K., and Rosati, A., The G.F.D.L. Modular Ocean Model Users Guide, GFDL Ocean Group Technical Report \# 2, 1993.

Perigaud, C.: Sea level oscillations observed with Geosat along the two shear fronts of the Pacific North Equatorial Countercurrent, J. Geophys. Res., 95, 7239-7248, 1990.

Philander, S. G. H.: Instabilities of Zonal Equatorial Currents, J. Geophys. Res., 81, 3725-3735, 1976.

Philander, S. G. H.: Instabilities of Zonal Equatorial Currents, 2, J. Geophys. Res., 83, 3679-3682, 1978.

Proehl, J. A.: The role of meridional flow asymmetry in the dynamics of tropical instability, J. Geophys. Res., 103, 24 597-24 618, 1998.

Qiao, L. and Weisberg, R. H.: Tropical instability wave kinematics: Observations from the Tropical Instability Wave Experiment, J. Geophys. Res, 100, 8677-8693, 1995.

Ripa, P.: General stability conditions for zonal flows ina one-layer model on the $\beta$-plane or the sphere, J. Fluid Mech., 126, 463489, 1983.

Ripa, P.: General stability conditions for a multi-layer model, J. Fluid Mech., 222, 119-137, 1991.

Seigel, A. D.: A comment on long waves in the Pacific Ocean, J. Phys. Ocean., 15, 1881-1883, 1985.

Semtner Jr., A. J. and Holland, W. R.: Numerical simulation of equatorial ocean circulation. Part I: A basic case in turbulent equilibrium, J. Phys. Ocean., 10, 667-693, 1980.

Stone, P. H.: On non-geostrophic baroclinic stability, J. Atmos. Sci., 23, 390-400, 1966.

Stone, P. H.: On non-geostrophic baroclinic stability: Part II, J. Atmos. Sci., 27, 721-726, 1970. 